\title{
Production of spectator hypermatter in relativistic heavy-ion collisions.
}

\author{
A.S. Botvina ${ }^{1,2}$, K.K. Gudima ${ }^{1,3}$, J. Steinheimer ${ }^{1}$, M. Bleicher ${ }^{1}$, and I.N. Mishustin ${ }^{1,4}$ \\ ${ }^{1}$ Frankfurt Institute for Advanced Studies, \\ J.W. Goethe University, D-60438 Frankfurt am Main, Germany \\ ${ }^{2}$ Institute for Nuclear Research, Russian Academy of Sciences, 117312 Moscow, Russia \\ ${ }^{3}$ Institute of Applied Physics, Academy of Sciences \\ of Moldova, MD-2028 Kishinev, Moldova and \\ ${ }^{4}$ Kurchatov Institute, Russian Research Center, 123182 Moscow, Russia
}

(Dated: October 29, 2018)

\begin{abstract}
We study the formation of large hyper-fragments in relativistic heavy-ion collisions within two transport models, DCM and UrQMD. Our goal is to explore a new mechanism for the formation of strange nuclear systems via capture of hyperons by relatively cold spectator matter produced in semi-peripheral collisions. We investigate basic characteristics of the produced hyper-spectators and evaluate the production probabilities of multi-strange systems. Advantages of the proposed mechanisms over an alternative coalescence mechanism are analysed. We also discuss how such systems can be detected taking into account the background of free hyperons. This investigation is important for the development of new experimental methods for producing hyper-nuclei in peripheral relativistic nucleus-nucleus collisions, which are now underway at GSI and are planned for the future FAIR and NICA facilities.

PACS numbers: 25.75.-q, 21.80.+a, 25.70.Mn
\end{abstract}




\section{INTRODUCTION}

Strange baryons (hyperons) were discovered in the 1950-s in reactions induced by cosmic rays. In nuclear reactions at high energies strange particles (baryons and mesons) are produced abundantly, and they are strongly involved in the reaction dynamics. The specifics of hypernuclear physics is that there is no direct experimental way to study hyperon-nucleon $(Y N)$ and hyperon-hyperon $(Y Y)$ interactions $(Y=\Lambda, \Sigma, \Xi, \Omega)$. When hyperons are captured by nuclei, hypernuclei are produced, which can live long enough in comparison with nuclear reaction times. Therefore, a nucleus may serve as a laboratory offering a unique opportunity to study basic properties of hyperons and their interactions. Double- and multi-strange nuclei are especially interesting, because they are more suitable for extracting information about the hyperon-hyperon interaction and strange matter properties.

The investigation of hypernuclei allows to answer many fundamental questions. Here we mention only some of them. First of all, studying the structure of hypernuclei helps to understand the structure of conventional nuclei too [1]. These studies lead to an extension of the nuclear chart into the strangeness sector [2, 3]. Second, hypernuclei provide a bridge between traditional nuclear physics (dealing with protons and neutrons) and hadron physics. Strangeness is an important degree of freedom for the construction of QCD motivated models of strong interactions [4]. And last but not least, strange particles are abundantly produced in nuclear matter at high densities, which are realized in the core of neutron stars [5]. The only way to describe realistically these physical conditions is to study the hyperon interactions in laboratory, and select theoretical models which pass the careful comparison with experimental data.

Traditionally, information about hypernuclear interactions is obtained from spectroscopy of hypernuclei combined with theoretical analyses. Production of kaons is often used for triggering hypernuclei, and by using kaon beams one can produce double hypernuclei. The theoretical studies are mainly concentrated on calculating the structure of nearly cold hypernuclei with baryon density around the nuclear saturation density, $\rho_{0} \approx 0.15 \mathrm{fm}^{-3}$. In this work we consider relativistic nucleus-nucleus collisions leading to copious production of hyperons and analyze new opportunities for hypernuclear physics realized in this case. As seen in experiments in the $\mathrm{GeV}$ domain of bombarding energies [6], $\Lambda$-hyperons are produced mainly in the participant zone, however, they have a broad rapidity distribution, so 
that a certain fraction of them can even be found in the spectator kinematical region. As shown by theoretical calculations using a coalescence model (see, e.g., [7]), some of these $\Lambda$ hyperons may be captured by nuclear spectator fragments produced in peripheral collisions. Indeed, experiments with light-ion beams at the LBL [8] and JINR [9] have demonstrated that hypernuclei can be formed in such reactions. The production of large excited spectator residues is well established in relativistic heavy-ion collisions also, see, e.g., Ref. [10]. At a later stage, these excited residues undergo de-excitation via evaporation, fission or multifragmentation [11]. Therefore, we expect that the capture of hyperons by spectators may lead to the formation of a big lump of excited matter containing a strangeness admixture. In the following, these excited spectators will break-up into conventional- and hyper-fragments [12].

We note that in central nucleus-nucleus collisions one can also produce light hyperfragments, as has been recently demonstrated in RHIC experiments [13]. However, because of the very high excitation energy released in the overlapping zone, it will be only possible to produce very light hypernuclei $(A \lesssim 4)$ in this way.

\section{MODELLING RELATIVISTIC NUCLEUS-NUCLEUS COLLISIONS}

\section{A. The DCM-QGSM approach}

One of the first models designed to describe the dynamics of energetic heavy-ion collisions was the intra-nuclear cascade model developed in Dubna [14]. In the following we refer to it as the Dubna Cascade Model (DCM). The DCM is based on the Monte-Carlo solution of a set of the Boltzmann-Uehling-Uhlenbeck relativistic kinetic equations with the collision terms, including cascade-cascade interactions. For particle energies below $1 \mathrm{GeV}$ it is sufficient to consider only nucleons, pions and deltas. The model includes a proper description of pion and baryon dynamics for particle production and absorption processes. In the original version the nuclear potential is treated dynamically, i.e., for the initial state it is determined using the Thomas-Fermi approximation, but later on its depth is changed according to the number of knocked-out nucleons. This allows one to account for nuclear binding. The Pauli principle is implemented by introducing a Fermi distribution of nucleon momenta as well as a Pauli blocking factors for scattered nucleons. 
At energies higher than about $10 \mathrm{GeV}$, the Quark-Gluon String Model (QGSM) is used to describe elementary hadron collisions [15, 16]. This model is based on the $1 / \mathrm{N}_{c}$ expansion of the amplitude for binary processes where $\mathrm{N}_{c}$ is the number of quark colours. Different terms of the $1 / \mathrm{N}_{c}$ expansion correspond to different diagrams which are classified according to their topological properties. Every diagram defines how many strings are created in a hadronic collision and which quark-antiquark or quark-diquark pairs form these strings. The relative contributions of different diagrams can be estimated within Regge theory, and all QGSM parameters for hadron-hadron collisions were fixed from the analysis of experimental data. The break-up of strings via creation of quark-antiquark and diquark-antidiquark pairs is described by the Field-Feynman method [17], using phenomenological functions for the fragmentation of quarks, antiquarks and diquarks into hadrons. The modified nonMarkovian relativistic kinetic equation, having a structure close to the Boltzmann-UehlingUhlenbeck kinetic equation, but accounting for the finite formation time of newly created hadrons, is used for simulations of relativistic nuclear collisions. One should note that QGSM considers the two lowest $\mathrm{SU}(3)$ multiplets in mesonic, baryonic and antibaryonic sectors, so interactions between almost 70 hadron species are treated on the same footing. This is a great advantage of this approach which is important for the proper evaluation of the hadron abundances and characteristics of the excited residual nuclei. The above noted two energy extremes were bridged by the QGSM extension downward in the beam energy [18].

In the course of a nucleus-nucleus collision strange particles are produced in both primary and secondary baryon and meson interactions $(\mathrm{B}+\mathrm{B} \rightarrow \mathrm{BYK}, \mathrm{M}+\mathrm{B} \rightarrow \mathrm{YK})$. The produced hyperons can propagate and re-scatter on other particles, and, occasionally, they may be located inside the projectile or target spectators. These hyperons can be absorbed by the spectators if their kinetic energy in the rest frame of the residual nucleus is lower than the attractive potential energy, i.e., the hyperon potential. In this case an exited residual system with nonzero strangeness will be formed. As known from previous studies the $\Lambda$-hyperon potential at the normal nuclear density $V_{\Lambda}\left(\rho_{0}\right)$ is around $-30 \mathrm{MeV}$. In our simulations we calculate the local nucleon density $\rho$ at the hyperon's position by taking into account only the nucleons in the vicinity of this hyperon, within a radius of $2 \mathrm{fm}$. This local density is then used to calculate the effective potential $V_{\Lambda}(\rho)$. Usually this potential is softer than the potential in normal nuclear matter, since spectators are quite dilute after primary interactions. 
The density dependence of this potential is parameterized following Ref. [19]:

$$
V_{\Lambda}(\rho)=-\alpha \frac{\rho}{\rho_{0}}\left[1-\beta\left(\frac{\rho}{\rho_{0}}\right)^{2 / 3}\right],
$$

where $\alpha=57.5 \mathrm{MeV}$, and $\beta=0.522$. In our calculations we follow the propagation of each $\Lambda$-hyperon during the whole reaction time, up to about $100 \mathrm{fm} / \mathrm{c}$, and the absorption criterion is checked regularly. After the absorption some surrounding nucleons may escape from the spectator, as a result of their interactions with hadrons. Therefore, $V_{\Lambda}(\rho)$ can decrease and the absorbed hyperon may become free, or, after new interactions, it may be captured again in another part of the spectator.

\section{B. The UrQMD approach}

For the investigation of spectator hypermatter formation in high energy heavy ion collisions we have also employed the Ultra-relativistic Quantum Molecular Dynamics model (UrQMD v2.3) [20, 21]. This non-equilibrium transport approach constitutes an effective solution of the relativistic Boltzmann equation. The underlying degrees of freedom are hadrons, and strings that are excited in energetic binary collisions. Mean fields can in principle be taken into account in this framework too, but for the present investigation we have run the model in the so called cascade mode without external potentials.

The nucleon's coordinates are initialized according to a Woods-Saxon profile in coordinate space and their momenta are assigned randomly according to the Fermi distribution in the rest frame of the corresponding nucleus. The hadrons are propagated on straight lines until the collision criterion is fulfilled: The collision takes place if the covariant relative distance $d_{\text {trans }}$ between two particles gets smaller than the distance $d_{0}$ corresponding to the total cross section $\sigma_{\text {tot }}$ (which depends on the energy $s$ and the kind of hadrons $h$ ),

$$
d_{\text {trans }} \leq d_{0}=\sqrt{\frac{\sigma_{\text {tot }}(\sqrt{s}, h)}{\pi}} .
$$

The reference frame that is used for the time ordering of the collisions is the C.M.-system of the nucleus-nucleus collision. However, each individual collision process is calculated in the rest frame of the binary collision.

In the UrQMD model 55 baryon and 32 meson species, ground state particles and all resonances with masses up to $2.25 \mathrm{GeV}$, are implemented with their specific properties and 
interaction cross sections. In addition, full particle-antiparticle symmetry is applied. Until now, isospin symmetry is assumed and only flavour-SU(3) states are taken into account. The elementary cross sections are calculated by detailed balance, or the additive quark model, or are fitted and parametrized according to the available experimental data. For resonance excitations and decays the Breit-Wigner formalism is employed assuming vacuum parameters.

Towards higher energies, the treatment of sub-hadronic degrees of freedom is of major importance. In the present model, these degrees of freedom enter via the introduction of a formation time for hadrons produced in the fragmentation of strings [22-24]. String formation and fragmentation is treated according to the Lund model. For hard collisions with large momentum transfer $(>1.5 \mathrm{GeV} / \mathrm{c})$ the Pythia model is used for the simulation of final states. The UrQMD transport model is successful in describing the yields and the $p_{t}$ spectra of various secondary particles in pp and pA collisions [25]. A compilation of results of the recent version UrQMD-2.3 compared to experimental data can be found in [26].

For our present purposes we have applied the following procedure: During the UrQMD calculational run, we check for all $\Lambda \mathrm{s}$ and $\Xi \mathrm{s}$ in time steps of $\Delta t=0.5 \mathrm{fm} / \mathrm{c}$, first selecting only particles which are in the rapidity interval $y_{b} \pm \Delta y$, where $y_{b}$ is the beam rapidity and $\Delta y=0.267$, the rapidity spread associated with the Fermi momenta of nucleons in the initial nucleus. For such particles the net baryon density in the local rest frame of the strange particle is then estimated. This is done by calculating the zero component of the net baryon current of all baryons within the same rapidity interval. For this purpose we assume that each baryon is represented by a Gaussian wave package of width $\sigma=1 \mathrm{fm}$ which is Lorentz contracted in the direction of motion.

For the hyperon absorption by spectators the same potential criterion as in DCM model was adopted: We calculate the kinetic energy of the strange particles, both $\Lambda$ and $\Xi$ hyperons, with respect to the rest frame of the projectile nucleus. Whenever this energy is less than the corresponding potential, calculated with Eq. (1), the particle is absorbed and removed from the cascade simulation. We have checked that the results do only weakly depend on the depth of the hyperon potential, when it is varied in a reasonable interval of $\pm 20 \%$. 


\section{FORMATION OF HYPERON-RICH SPECTATOR MATTER}

A detailed picture of peripheral relativistic heavy-ion collisions has been established in many experimental and theoretical studies. Nucleons from the overlapping parts of the projectile and target (participant zone) interact intensively between themselves and with other hadrons produced in primary and secondary collisions. Nucleons from the non-overlapping parts do not interact strongly, and they form the residual nuclear systems, which we call spectators. In all transport models the production of hyperons is associated with nucleonnucleon collisions, e.g., $\mathrm{p}+\mathrm{n} \rightarrow \mathrm{n}+\Lambda+\mathrm{K}^{+}$, or collisions of secondary mesons with nucleons, e.g., $\pi^{+}+\mathrm{n} \rightarrow \Lambda+\mathrm{K}^{+}$. Strange particles are mainly produced in the participant zone, however, they can re-scatter and populate the whole momentum space around the colliding nuclei.

In the present paper we are interested mostly in hyperons which propagate with velocities close to the initial velocities of the nuclei, i.e., in the vicinity of nuclear spectators. We assume that such hyperons can be absorbed by the spectators if their kinetic energy (in the rest frame of the spectator) is lower than the potential generated by neighbouring spectator nucleons. Due to the secondary interactions these spectators are excited and have a dilute (subnuclear) density. As was previously investigated in detail [10, 27, 28], the further evolution of the spectators depends on their excitation energy. At lower excitation energies (below $2 \div 3 \mathrm{MeV}$ per nucleon) they de-excite via evaporation and/or fission-like processes, while at higher excitation energies they undergo multifragmentation [11]. We expect the same behaviour for the residual nuclei containing hyperons.

As was also found in previous studies of spectator fragmentation [27], there exists a strong correlation between the excitation energy transferred to the spectator matter and the number of high energy particles produced in the participant zone. On the other hand, the multiplicity of strange particles is also proportional to the number of participants. Therefore, we expect that the hyper-spectators will be very excited, and finally they will break-up into many fragments, some of them will contain the captured hyperons. This process was already analysed in Ref. [12].

The DCM model gives a very good description of yields and spectra of free hyperons and kaons produced in relativistic heavy-ion collisions. For example, in Fig. 1 the calculated inclusive rapidity distributions of $\Lambda$ and $\Sigma^{0}$-hyperons are compared with experimental data 
of E896 experiment at AGS [29]. As one can clearly see, the primary nucleon-nucleon

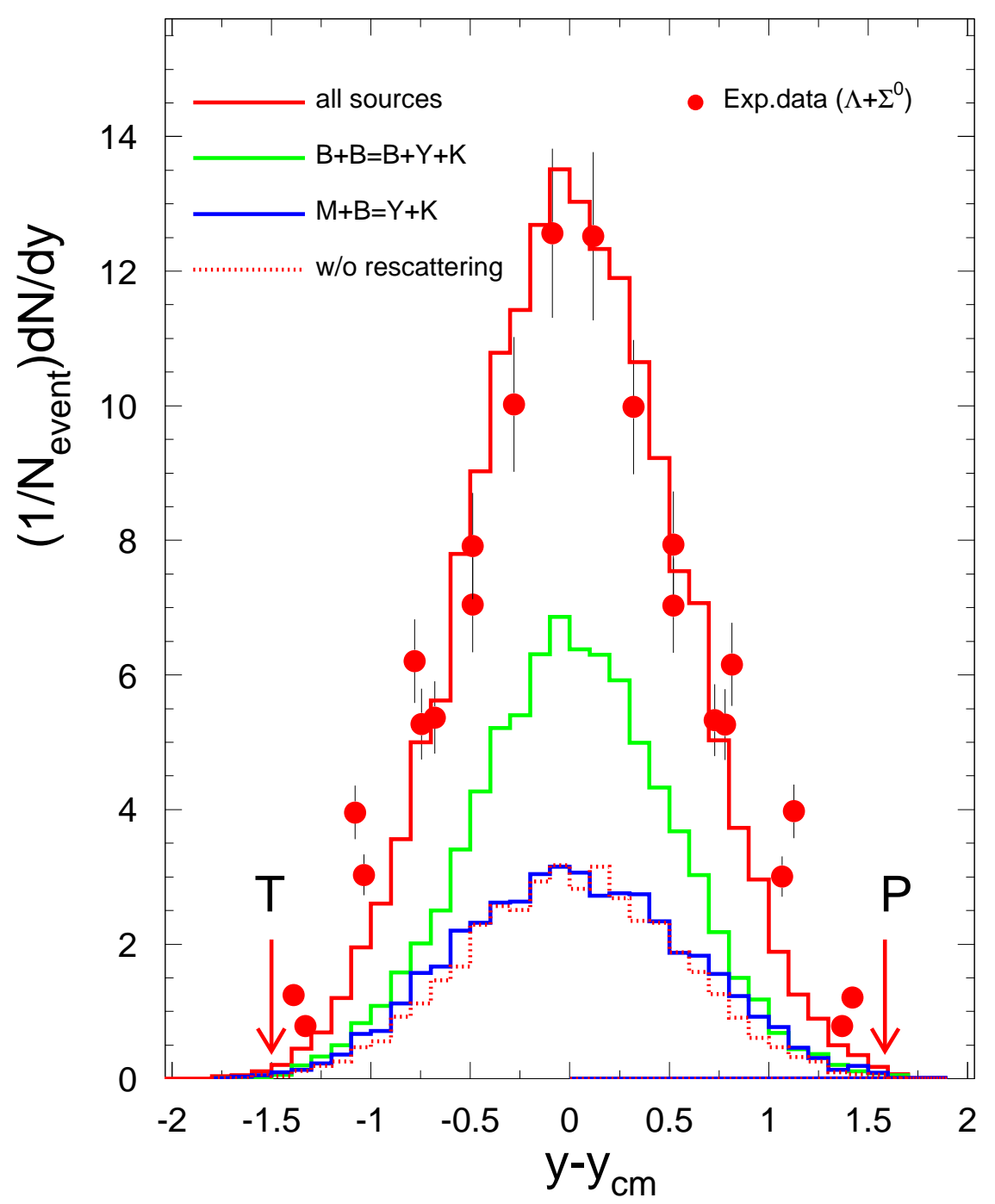

FIG. 1: Rapidity distribution of $\Lambda$ and $\Sigma^{0}$ hyperons produced in collisions of Au projectile with momentum $11 \mathrm{GeV} / \mathrm{c}$ per nucleon on Au target. Red circles are experimental data [29]. Histograms show contributions of different processes as predicted by DCM. Rapidities of the projectile (P) and target $(\mathrm{T})$ are shown by arrows.

interactions, which are noted as 'without rescattering', are responsible only for a small fraction of the strange particles. The secondary interactions of baryons and mesons provide the dominant contribution to the production of hyperons at all rapidities, and they are 
crucially important for description of the data. Similarly, the UrQMD model proved to be very successful in description of the strangeness production in high-energy nuclear collisions (see, e.g., [26]).

Since both the DCM and UrQMD can follow the evolution of all interacting particles in space and time, we can predict where and when the produced $\Lambda$ is absorbed in a projectile or target spectator. As a first example, in Fig. 2 we demonstrate the spatial distribution of $\Lambda$-hyperons, which satisfy the capture criterion described above. This figure shows the coordinates of absorption points projected into the transverse plane ( $\mathrm{X}$ and $\mathrm{Y}$ coordinates), perpendicular to the beam axis ( $\mathrm{Z}$ coordinate). The results are presented for $\mathrm{Au}+\mathrm{Au}$ collisions at a beam energy of $20 \mathrm{GeV}$ per nucleon and an impact parameter of $8.5 \mathrm{fm}$. Such collisions will be studied in future experiments at FAIR (Darmstadt, Germany) and at NICA (JINR, Dubna, Russia). The figure accumulates the simulation results after $2 \cdot 10^{5}$ collision events and integrated over the reaction time. For a better resolution of the scatter plot we show only about $10 \%$ of the absorption points taken randomly. Only DCM results are presented, since the UrQMD gives the same qualitative predictions.

In this plot and the following figures we show the elementary hadron interactions which are responsible for the production of the absorbed $\Lambda$-hyperons. This information is important to understand the physics of the absorption process. One can see that the contribution of direct nucleon-nucleon and pion-nucleon interactions is rather small, since the kinetic energy of produced hyperons is quite high in the rest frame of the nuclear residues. Only few hyperons emerging from these interactions may be captured by spectators. The largest number of absorbed hyperons is produced in the secondary interactions of strange particles with nucleons, e.g., in strangeness exchange reactions induced by antikaons and in the rescattering of fast hyperons on slow spectator nucleons.

It is instructive to investigate the absorption rate as a function of time to extract the coordinates of absorption points along the beam axis. The correlation between the time and $\mathrm{X}-\mathrm{Z}$ coordinates of the absorption points is illustrated in Fig. 3. One can see that some absorption events happen at a very early time $(\lesssim 10 \mathrm{fm} / \mathrm{c})$, when hyperons produced in the hot participant are captured by a few nucleons located in this zone. However, the contribution of this mechanism to the total capture yield is rather small. Moreover, due to intensive interactions taking place in the participant zone, many of the surrounding nucleons will be kicked out at later stages and the hyperons can become free again. This is why in 
the DCM calculations we have introduced a multiple check of the absorption criterion at later time steps.

After the first $10 \mathrm{fm} / \mathrm{c}$, when the projectile and target residues are completely separated in coordinate space, the capture process is entirely associated with the interactions of strange particles in the non-overlapping zones of colliding nuclei. Reactions induced by antikaons and hyperons with velocities close to projectile and target velocities provide the dominant contribution. One can see from Fig. 3 that this process continues for tens of fm/c, and it is quite possible to produce a hypernuclear residue even at the time of around $50 \mathrm{fm} / \mathrm{c}$. By looking at the $\mathrm{X}$-coordinates of the absorption points one can clearly see that the region of the $\Lambda$ absorption moves from the overlapping zone at early time to the non-overlapping spectator parts for later times, as a result of many secondary interactions.

The space-integrated time evolution of the $\Lambda$ absorption is presented in Fig. 目 for the same $\mathrm{Au}+\mathrm{Au}$ collisions at $20 \mathrm{~A} \mathrm{GeV}$ projectile energy. For convenience, the capture rate is normalized to the total number of collision events. This figure allows for a quantitative estimate of the absorption rates associated with different interaction channels. From the DCM results one can see that about $90 \%$ of $\Lambda$ s absorbed in spectators come from secondary reactions when previously produced hyperons re-scatter on spectator nucleons. After the first $50-70 \mathrm{fm} / \mathrm{c}$ the absorption rate becomes low and it drops exponentially, signalling that the dynamical process is coming to an end, and the equilibrium stage of the spectator's evolution should be considered.

One can notice a difference between the two considered models: the absorption rate decreases faster in the UrQMD model. The reason could lie in the treatment of $\Lambda$ absorption at early times: In the UrQMD $\Lambda$-hyperons are considered as finally absorbed after the capture criterion is first satisfied. No further propagation of these hyperons is simulated. As we have mentioned, in the DCM we take into account a time evolution of the nucleon density in the vicinity of the captured hyperon. Some nucleons can leave this neighbourhood at a later time, leading to a decrease of the local density so that the hyperon may escape. This happens mostly at the early stage $(\lesssim 10 \mathrm{fm} / \mathrm{c})$. On the other hand, at later times some of these hyperons may be captured again in the spectator zone, and this effect will increase the absorption rate. Therefore, the absorption process is longer in the DCM as compared with the UrQMD. However, in the end these two dynamical scenarios of hyperon absorption give very similar integrated yields. 


\section{CHARACTERISTICS OF SPECTATOR HYPER-MATTER.}

As one can see in Fig. 4, 80-90\% of the $\Lambda$ s are absorbed in spectators by the time of $80 \mathrm{fm} / \mathrm{c}$. At this stage all secondary interactions are practically over and we can determine the parameters of the spectator residues. Figure 5 shows the time evolution of the average mass number of spectators as predicted by DCM and UrQMD calculations. The decrease of masses with time is obviously caused by secondary interactions of fast particles with spectator nucleons. In the UrQMD a nucleon is defined as spectator if it has not participated in elementary interactions. On the other hand, the DCM definition of spectators includes nucleons which underwent interactions and have been recaptured by the nuclear potential. By this reason the DCM spectator residues are bigger. It is seen that the total number of nucleons in the spectators is nearly saturated after $70 \mathrm{fm} / \mathrm{c}$. During the dynamical stage the high energy neutrons and protons interact similarly, therefore, the average neutron-to-proton ratio in the spectators is almost the same as in colliding nuclei.

These spectator residues acquire some excitation energy, which can be calculated within both models too. However, it is well known from experiments with conventional nuclear matter that in relativistic collisions the average temperature of such residues is saturated at the level of 5-6 MeV, despite of large event-by-event fluctuations of the excitation energy

[11, 30]. We expect similar average temperatures in hyper-residues too, especially when the number of captured hyperons is much smaller than the number of nucleons.

One important practical result of this study is the estimated probability for producing spectator residues with different numbers of captured $\Lambda \mathrm{s}$. These probabilities are shown in Fig. 6] for residues containing up to $3 \Lambda$ hyperons, together with their mean masses, for $\mathrm{Au}$ $+\mathrm{Au}$ and $\mathrm{p}+\mathrm{Au}$ collisions with energies of 2 and $20 \mathrm{~A} \mathrm{GeV}$. The chosen beam energies correspond to the present GSI experiments, and the future FAIR experiments. The calculations were performed with minimum bias, i.e., integrated over all impact parameters. For each reaction $10^{6}$ Monte-Carlo events were generated. According to the DCM calculation, at the highest energy the probability for capturing one hyperon in the spectator amounts to a few percents, and two hyperons may be captured with a significant probability of about $\sim 5 \cdot 10^{-4}$. Furthermore, even $3 \Lambda$-hyperons may be captured by spectators with a low but measurable probability $\sim 10^{-5}$. The absorption of a higher number of hyperons is also feasible. This new mechanism opens a unique opportunity to produce and study multi-strange 
systems, which are not conceivable in other nuclear reactions. Since the predicted masses of the hyper-spectators are quite large, one can even speak about the formation of excited hypermatter. We have found that variation of the hyperon potential within a reasonable range does only weakly influence the capture rates at the highest energy, because these hyperons undergo multiple rescattering and de-accelerate considerably in the spectator matter.

Compared to the DCM results, the probabilities for producing hyper-spectators predicted by the UrQMD model can differ by a factor 2 . This difference shows the uncertainty in the calculations based on the best theoretical models available at present. Still the qualitative agreement between the two transport models gives us a confidence that this new method can be successfully used for producing new hypernuclei.

From calculations with the UrQMD model we have also estimated the probability of a $\Xi$ being absorbed in the spectator fragment at $E_{l a b}=20 \mathrm{~A} \mathrm{GeV}$ to be on the order of $10^{-5}$ per event. Such an absorption would eventually lead to the formation of $\Xi$-hypernuclei in future experiments. This opens the interesting possibility to study a new multi-strange system which is even less understood as the conventional $\Lambda$-hypernuclei.

For completeness, in Fig. 7 we show the DCM predictions for the mass distributions of residues (representing both conventional matter and hyper-matter), integrated over all impact parameters. One can notice a significant difference in the shape: for conventional residues the distribution is very broad, and it has a sharp peak around the projectile mass, corresponding to very peripheral collisions. These heavy residuals are weakly excited since only a few nucleons participated in the reaction. On the contrary, when hyperons are absorbed many nucleons are involved in secondary interactions, and some of them leave the spectators. As a result, it is very unlikely to produce hyper-residues with masses around the initial mass in heavy-ion collisions. However, the distribution remains very broad and practically all intermediate-mass residues can be produced with considerable probability.

We should note that the formation of hyper-residues was earlier discussed in Ref. [31] for the reactions initiated by protons. As one can see from Fig. 6 the probabilities for production of multi-strange hyper-residues in such reactions are lower than in nucleus-nucleus collisions. This is easy to understand, since more hyperons in the spectator region can be produced when more particles participate in the interactions. However, the channels with absorption of one $\Lambda$, and even with two $\Lambda$ s at the highest energy can be still very probable in $\mathrm{p}+\mathrm{Au}$ collisions. In addition, the masses of hyper-residues will be larger, since in this case less 
target nucleons are involved in the reaction. The main problem expected for proton beams is that experimentally it is very difficult to identify decay products of a slow hyper-nucleus in a background of free $\Lambda$ decays. Relativistic heavy-ion collisions have essential advantages: Because of the Lorentz factor their lifetime becomes longer and projectile hyper-fragments can travel a longer distance. This makes possible to use sophisticated vertex detectors and fragment separation technique for their identification.

\section{RAPIDITY DISTRIBUTIONS OF HYPER-RESIDUES AND FREE $\Lambda$ - HYPERONS}

For the experimental identification of hypernuclei it is of crucial importance to know the background associated with free hyperons produced in a reaction. For example, let us consider the reaction ${ }^{6} \mathrm{Li}+{ }^{12} \mathrm{C}$ at a beam energy of $1.9 \mathrm{GeV}$ per nucleon, which was employed on the first stage of the HypHi experiment at GSI [32]. Figure 8] shows the rapidity distributions of the free and captured $\Lambda$ s produced in this reaction. One can see that for these light nuclei the yield of free $\Lambda$ s in the spectator region is 1-2 orders of magnitude larger than the yield of hypernuclei originating from the projectile spectator. For this reason, the fraction of pions coming from the decay of hypernuclei is very small and careful correlation measurements are required to identify these hypernuclei. It is interesting that this fraction is higher for the larger target spectator, however, in the experiment only projectile fragments can be detected.

A higher ratio of spectator hypernuclei to free $\Lambda$ s is obtained in reactions involving heavy nuclei. Figure 9 presents the result of the DCM calculation for $\mathrm{Au}+\mathrm{Au}$ collisions at a beam energy of $20 \mathrm{~A} \mathrm{GeV}$, as planned in future FAIR experiments. One can see clearly that the excess of free $\Lambda$ s over hypernuclei in spectator kinematic zones is minimal in this case, i.e., less than a factor $2 \div 3$. This significant improvement has a simple explanation: More secondary hyperons interact with spectator nucleons at a later time of the reaction $(t>20$ $\mathrm{fm} / \mathrm{c})$. These hyperons have velocities close to the velocities of spectators, therefore, it is more probable for them to have other spectator nucleons nearby. 


\section{MOMENTUM DISTRIBUTION OF ABSORBED HYPERONS.}

As follows from the potential criterion (see Section II) the absorbed $\Lambda$ hyperons have small momenta in the rest frame of the spectators. Direct DCM and UrQMD calculations of the invariant momentum distribution of the absorbed $\Lambda \mathrm{s}$ for $\mathrm{Au}(20 \mathrm{~A} \mathrm{GeV})+\mathrm{Au}$ collisions are shown in Fig. 10. One can see that the distribution is rather narrow with a smooth cut-off for hyperon momenta greater than $250 \div 300 \mathrm{MeV} / \mathrm{c}$. However, this distribution is not step-like, and we can even approximate the slope of the spectrum at $P>100 \mathrm{MeV} / \mathrm{c}$ by a Boltzmann distribution with apparent temperature $T \approx 10 \mathrm{MeV}$. This low temperature indicates that these hyperons are rather slow in the considered reference frame, and this is a typical temperature for particles coming from the spectator matter as was demonstrated long ago by the analysis of ALADIN data [27].

Sometimes the coalescence criterion for cluster formation is used, when a hyperon is considered to be absorbed by a nuclear cluster if they are close in momentum and coordinate space. Our results shown in Fig. 10 justify using the coalescence criterion in a simple consideration, when secondary interactions in spectators are neglected. Recently the GiBUU model [7] was generalized to describe hypernuclei production in similar relativistic nucleusnucleus collisions. They have calculated excited residues after the dynamical stage. Then the SMM [11] was applied to model the de-excitation and multifragmentation of these residues into conventional fragments. Afterwards, a coalescence prescription to form hyperfragments from these conventional fragments and free $\Lambda$ s was applied. An important feature of our approach is the assumption that the absorption takes place fastly (during first few tens of $\mathrm{fm} / \mathrm{c}$ ) in excited residues before their break-up. The de-excitation itself takes a considerable time, of about $10^{2}-10^{4} \mathrm{fm} / \mathrm{c}$ depending on excitation energy. Therefore, the final yields of specific hypernuclei in our approach will be different from the approach of Ref. [7].

Nevertheless, the coalescence model can be successfully used to take into account the final state interaction between free nucleons leading to the formation of lightest clusters, as was shown already in Ref. [14]. We expect that this mechanism may work in the case of light spectators and free hyperons too. Similar results concerning the production of light hyper-fragments can be obtained within the thermal models [33-35]. A connection between parameters of coalescence and thermal models describing the production of lightest clusters at high excitation energies is under discussion for a long time, see, e.g., Refs. [36, 37]. 
In principle, the coalescence prescription can be applied for free nucleons and hyperons to investigate the formation of both, conventional light nuclei (such as $d, t, H e$ ), and light hypernuclear fragments produced in the spectator region. However, our preliminary calculations for $\mathrm{Au}+\mathrm{Au}$ collisions have shown that a probability for the production of hyper-clusters by this mechanism is by several orders of magnitude smaller than the absorption of hyperons in the spectators, as considered in this paper. On the other hand, we have found that the coalescence mechanism is more efficient for the formation of hyper-clusters in the midrapidity zone [38].

\section{DISINTEGRATION OF HOT SPECTATOR MATTER INTO HYPER- FRAGMENTS.}

In the final stage of the reaction the excited hyper-residues undergo de-excitation. At low excitation energy, this should be an evaporation (and, may be, fission) process, similar to what is well known for the conventional nuclei. However, as we see from our calculations and also from analyses of experimental data [11, 27, 28], the thermalized residual nuclei have rather high excitation energies in the nuclear scale, and, consequently, they should undergo multifragmentation with a characteristic time of about $100 \mathrm{fm} / \mathrm{c}$. The generalization of the Statistical Multifragmentation Model (SMM) [11] into the strangeness sector by including $\Lambda$-hyperons has been done in Ref. [12]. It was demonstrated that the fragment mass distributions are quite different for fragments with different strangeness content. This means that the multifragmentation of excited hypernuclear systems proceeds in a different way as compared with conventional nuclei. The reason is the additional binding energy of hyperons in nuclear matter. It was also shown that the yields of fragments with two $\Lambda$ s depend essentially on the mass formulae (i.e., on details of $\Lambda N$ and $\Lambda \Lambda$-interactions) used for the calculations [12, 39]. Therefore, an analysis of double hypernuclei can help to improve these mass formulae and reveal information about the hyperon-hyperon interaction. In Ref. [40] the decay of light excited hyper-systems was considered within the framework of the Fermi break-up model. It was also concluded that the production rate of single and double hyper-nuclei is directly related to their binding energy.

We would like to mention another canonical statistical model [41], which was developed

in line with [12]. It gives similar results concerning hypernuclei produced after the decay of 
excited hyper-systems. A detailed analysis of the de-excitation process taking into account the primary dynamical stage of the reaction will be presented in a subsequent publication.

\section{CONCLUSION}

Within the DCM and UrQMD models we have investigated the production of hyperons in peripheral relativistic heavy ion collisions and their capture by the attractive potential of spectator residues. Contrary to the coalescence mechanism, which may be responsible for the formation of light hyperfragments, this capture process can also lead to the production of heavy hypernuclei. Yields of such hypernuclei, are quite significant, and a very broad mass distribution of hypernuclear spectators can be obtained. An important advantage of this method over the traditional reactions induced by kaons is that it provides a natural way to produce multi-hyperon systems. As our calculations show, the relative probabilities to produce residual nuclei with $2 \Lambda$ and $3 \Lambda$ in $\mathrm{Au}+\mathrm{Au}$ reaction at $20 \mathrm{~A} \mathrm{GeV}$ are about $5 \cdot 10^{-4}$ and $10^{-5}$, respectively. These probabilities should be sufficient for systematic studies of such systems in the future FAIR and NICA experiments.

Conventional spectator matter after relativistic nuclear collisions was intensively investigated during the last 20 years [11, 30]. Similarly, we expect the production of excited hypermatter at a baryon density $\rho \sim 0.1-0.8 \rho_{0}$ and temperatures around $T \sim 3-7 \mathrm{MeV}$, which are typical for the coexistence region of the nuclear liquid-gas phase transition. This opens the possibility to study this phase transition in nuclear matter with a strangeness admixture, that is important for the physics of neutron stars. An analysis of the disintegration of this matter into cold non-strange fragments and hyper-fragments will reveal information about the properties of hypernuclei, their binding energies, and, finally, $Y N$ and $Y Y$ interactions. Hypernuclei of all sorts are expected to be produced in his way, including nuclei around drip lines, which is not possible with traditional methods.

It would be very helpful to perform further detailed theoretical calculations, which include all stages of the reaction: 1) production of hyperon-rich spectator nuclear systems, 2) formation of hyper-fragments via decay of these systems, 3) decay of the hypernuclei via weak interaction with production of non-strange particles in the final state. The DCM and UrQMD approaches can be used for the description of the dynamical stage and they can be followed by statistical models, e.g., as the SMM. In this case all products of the reaction 
can be determined on an event-by-event basis. Advanced correlation measurements, which are necessary to identify hypernuclei, can be tested with these models too.

The detection of spectator hyper-nuclei may suffer from a background problem, which may require sophisticated experimental methods to solve. However, it has already been demonstrated experimentally that in peripheral collisions of light projectiles and targets the cross section for the capture of produced $\Lambda$-hyperons by projectile residues may be of the

order of a few microbarns [9], and this cross section allows for an experimental identification of the produced hypernuclei [32]. As we have demonstrated, involving heavy projectiles and targets can indeed improve the signal-to-background ratio significantly. We believe that hypernuclear physics will benefit strongly from exploring new production mechanisms and the novel detection technique associated with spectator fragmentation reactions.

\section{Acknowledgments}

This work was supported by BMBF, HGS-HIRe and the Hessian LOEWE initiative through the Helmholtz International center for FAIR (HIC for FAIR). We acknowledge also partial support from the DFG grant 436 RUS 113/957/0-1, as well as the grants RFBR 09-02-91331 and NSH-7235.2010.2 (Russia). K.K.G. thanks FIAS for the hospitality.

[1] O. Hashimoto, H. Tamura, Prog. Part. Nucl. Phys. 57, 564 (2006).

[2] J. Schaffner, C.B. Dover, A. Gal, C. Greiner, and H. Stoecker, Phys. Rev. Lett. 71, 1328 (1993).

[3] W. Greiner. J. Mod. Phys. E5, 1 (1995).

[4] P. Papazoglou, S. Schramm, J. Schaffner-Bielich, H. Stoecker, and W. Greiner, Phys. Rev. C $\mathbf{5 7}, 2576(1998)$

[5] J. Schaffner and I.N. Mishustin, Phys. Rev. C 53, 1416 (1996).

[6] X. Lopez et al., Prog. Part. Nucl. Phys. 53, 149 (2004).

[7] Th. Gaitanos, H. Lenske, and U. Mosel, Phys. Lett. B 663, 197 (2008); ibid., Phys. Lett. B 675, 297 (2009).

[8] K.J. Nield et al., Phys. Rev. C 13, 1263 (1976). 
[9] S. Avramenko et al., JETP Lett. 48, 516 (1988);

S. Avramenko et al., Nucl. Phys. A 547, 95c (1992).

[10] A.S. Botvina et al., Nucl. Phys. A 584, 737 (1995).

[11] J.P. Bondorf, A.S. Botvina, A.S. Iljinov, I.N. Mishustin, and K. Sneppen, Phys. Rep. 257, 133 (1995).

[12] A.S. Botvina and J. Pochodzalla, Phys. Rev. C 76, 024909 (2007).

[13] The STAR collaboration, arXiv:1003.2030 [nucl-ex]

[14] V.D. Toneev, K.K. Gudima, Nucl. Phys. A 400, 173c (1983).

[15] V.D. Toneev, N.S. Amelin, K.K. Gudima, S.Yu. Sivoklokov, Nucl. Phys. A 519, 463c (1990).

[16] N.S. Amelin, E.F. Staubo, L.S. Csernai et al., Phys.Rev. C 44, 1541 (1991).

[17] R.D. Field, R.P. Feynman, Nucl. Phys. B 136, 1 (1978).

[18] N.S. Amelin, K.K. Gudima, S.Yu. Sivoklokov, V.D. Toneev, Sov. J. Nucl. Phys. 52, 272 (1990).

[19] I. Ahmad, M. Mian and M. Z. Rahman Khan, Phys. Rev. C 31, 1590 (1985).

[20] M. Bleicher et al., J. Phys. G 25, 1859 (1999).

[21] S. A. Bass et al., Prog. Part. Nucl. Phys. 41, 255 (1998).

[22] B. Andersson, G. Gustafson and B. Nilsson-Almqvist, Nucl. Phys. B 281, 289 (1987).

[23] B. Nilsson-Almqvist and E. Stenlund, Comput. Phys. Commun. 43, 387 (1987).

[24] T. Sjostrand, Comput. Phys. Commun. 82, 74 (1994).

[25] E. L. Bratkovskaya et al., Phys. Rev. C 69, 054907 (2004).

[26] H. Petersen, M. Bleicher, S. A. Bass and H. Stocker, arXiv:0805.0567 [hep-ph].

[27] H. Xi et al., Z. Phys. A 359, 397 (1997).

[28] R.P. Scharenberg et al., Phys. Rev. C 64, 054602 (2001).

[29] S.Albergo et al., Phys. Rev. Lett. 88, 062301 (2002).

[30] J. Pochodzalla, Prog. Part. Nucl. Phys. 39, 443 (1997).

[31] Z.Rudy, W.Cassing et al., Z. Phys. A 351, 217 (1995).

[32] The HypHI collaboration:

Letter of Intent (2005), http://www.gsi.de/documents/DOC-2005-Feb-432-2.pdf;

Progress Report (2006), http://www.gsi.de/documents/DOC-2006-Jan-2-1.pdf.

[33] P. Braun-Munzinger, J. Stachel, J. Phys. G G21, L17 (1995).

[34] J. Steinheimer, M. Mitrovski, T. Schuster, H. Petersen, M. Bleicher, H. Stoecker, Phys. Lett. B 676, 126 (2009). 
[35] A.Andronic, P.Braun-Munzinger, J.Stachel, H.Stoecker, Phys. Lett. B 697, 203 (2011).

[36] A. Mekjian, Phys. Rev. Lett. 38, 640 (1977).

[37] W.Neubert and A.S.Botvina, Eur. Phys. J. A 17, 559 (2003).

[38] K.K.Gudima, et al., work in progress. .

[39] C.Samanta, A.S.Botvina, I.N.Mishustin, and W.Greiner, work in progress.

[40] A.S. Lorente, A.S. Botvina, and J. Pochodzalla, Phys. Lett. B 697, 222 (2011).

[41] S.Das Gupta, Nucl. Phys. A 822, 41 (2009); V.Topor Pop, S.Das Gupta, Phys. Rev. C 81, $054911(2010)$. 


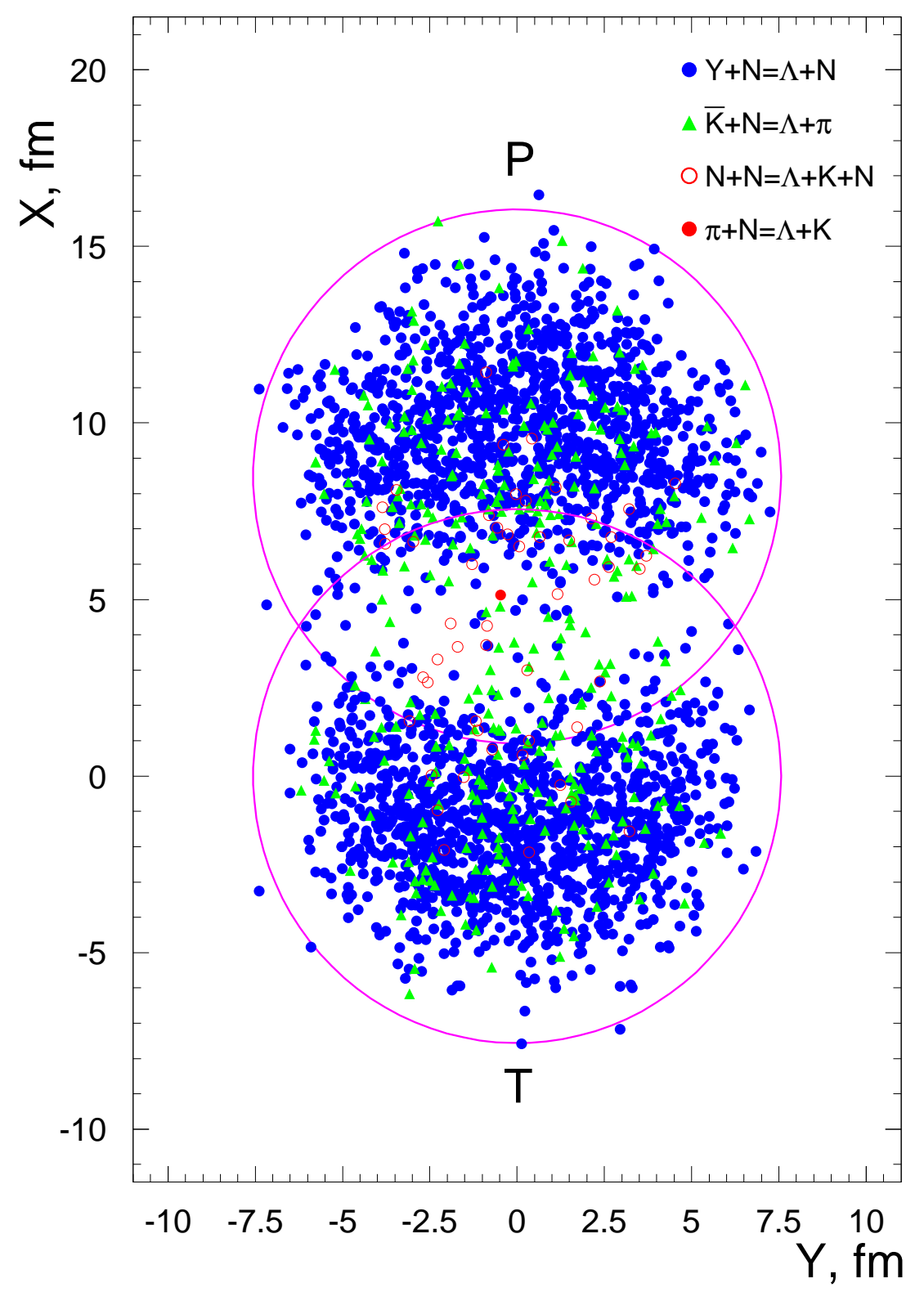

FIG. 2: Coordinates of $\Lambda$ absorption points projected into the transverse plane perpendicular to the beam axis. Results of DCM calculations are shown for $\mathrm{Au}(20 \mathrm{~A} \mathrm{GeV})+\mathrm{Au}$ collisions with an impact parameter of $8.5 \mathrm{fm}$. The circles are outer contours of projectile $(\mathrm{P})$ and target $(\mathrm{T})$ nuclei at this impact parameter Processes creating the absorbed hyperon, such as interactions of secondary hyperons, antikaons, nucleons and pions with nucleons, are indicated by different symbols. 


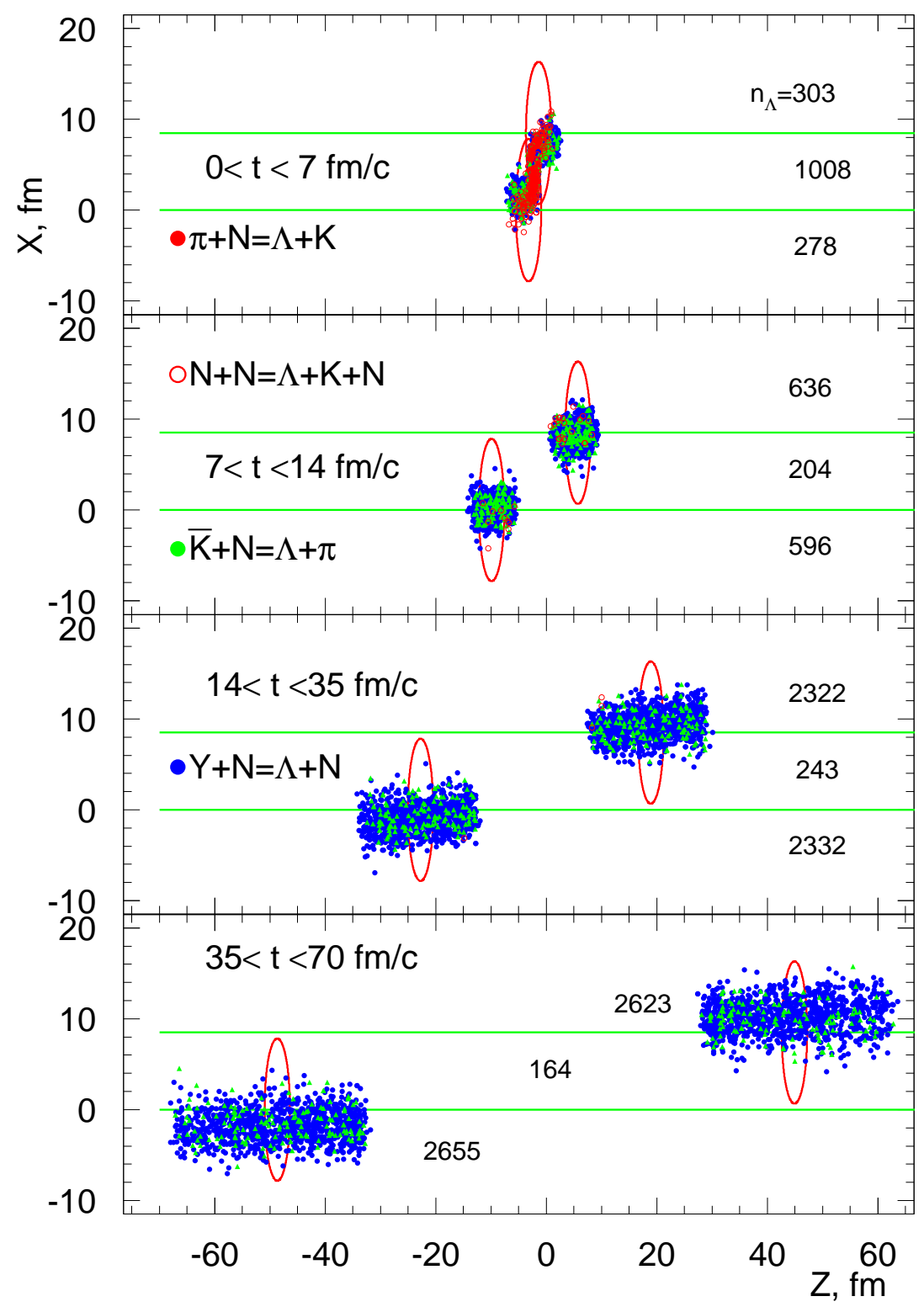

FIG. 3: The same DCM calculations with symbol notations as in Fig. 2, but in the $\mathrm{X}-\mathrm{Z}$ plane. The symbols show the coordinates of $\Lambda$ absorption points in projectile and target spectators. Ellipses show the average positions of projectile and target nuclei during time intervals indicated in the figure (from top to bottom) in the system of equal velocities. Number of hyperons $n_{\Lambda}$ (per $2 \cdot 10^{5}$ events) captured in the participant and spectator zones during these intervals are noted on the right side. 


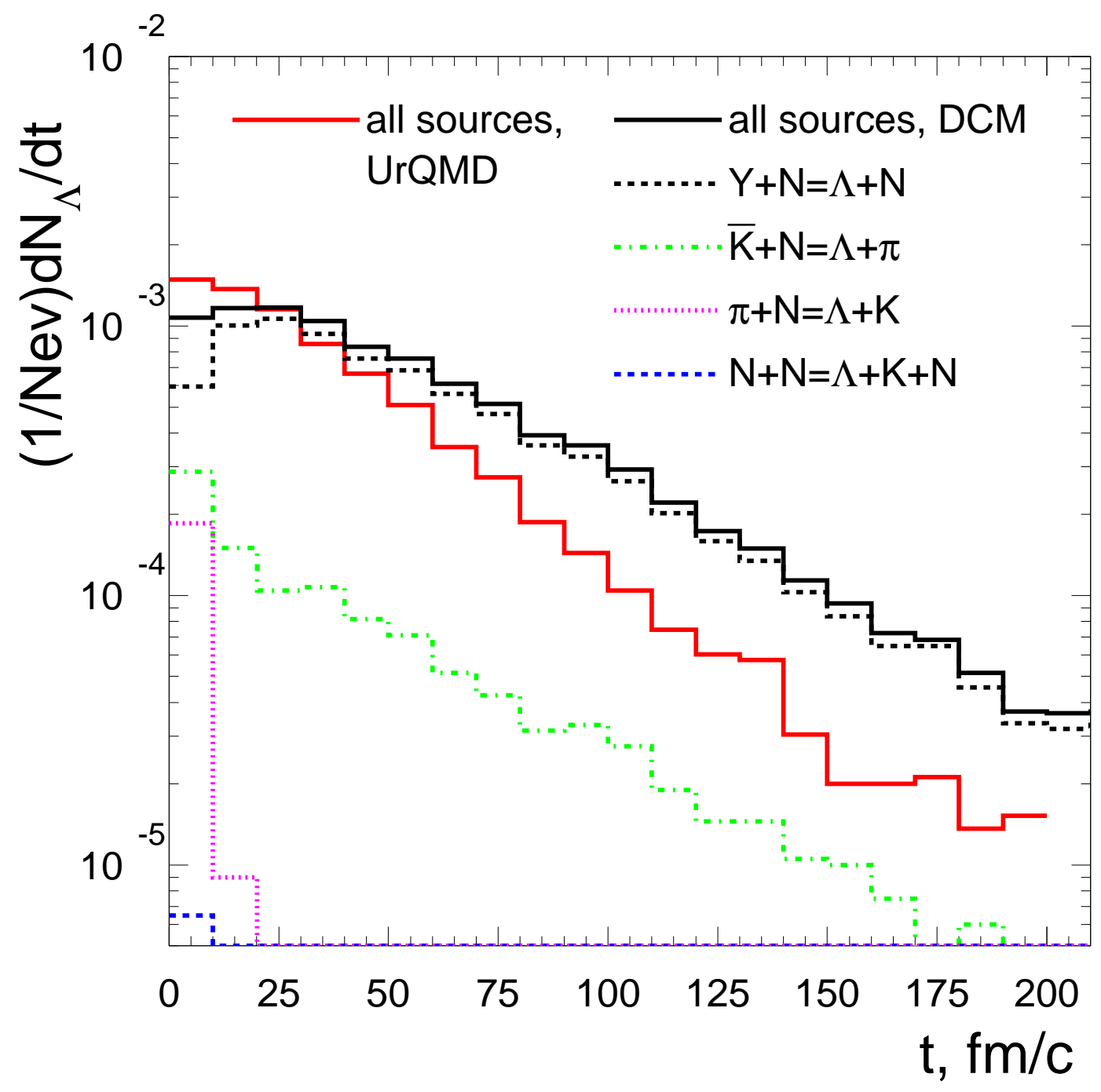

FIG. 4: The rate of the $\Lambda$ absorption by spectators (versus time) as calculated in DCM and UrQMD model for $\mathrm{Au}(20 \mathrm{~A} \mathrm{GeV})+\mathrm{Au}$ reaction. Black, green, blue and violet lines give the total rate and contributions of specific reactions to production of these $\Lambda$ as predicted by DCM. Red line is the total rate predicted by UrQMD. 


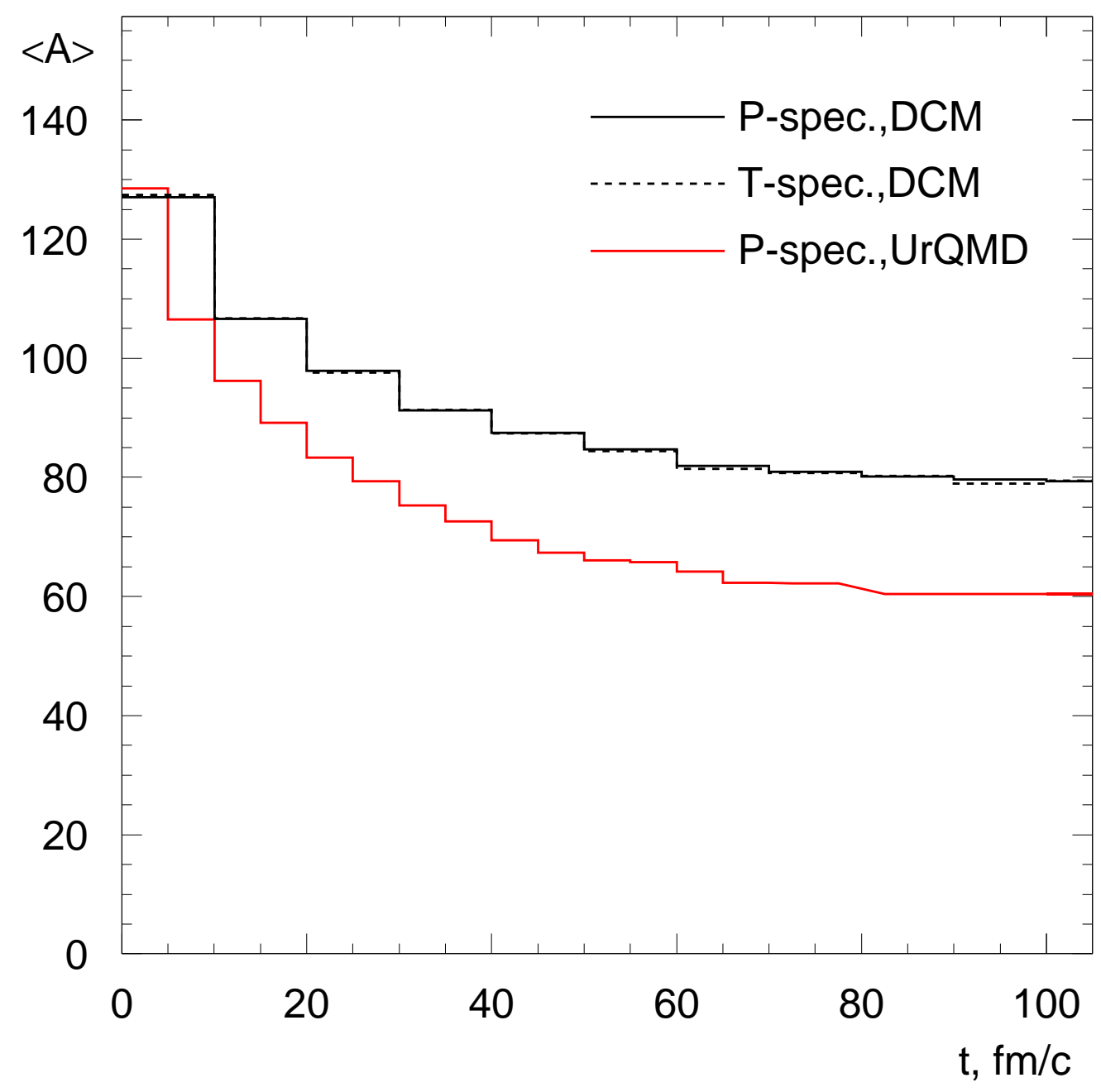

FIG. 5: Time evolution of the mean number of nucleons in the spectator $(\mathrm{P}-$ projectile, $\mathrm{T}-$ target) residual nuclei after $\Lambda$ absorption as predicted by the DCM and UrQMD calculations. The reaction is as in Fig. 2, 

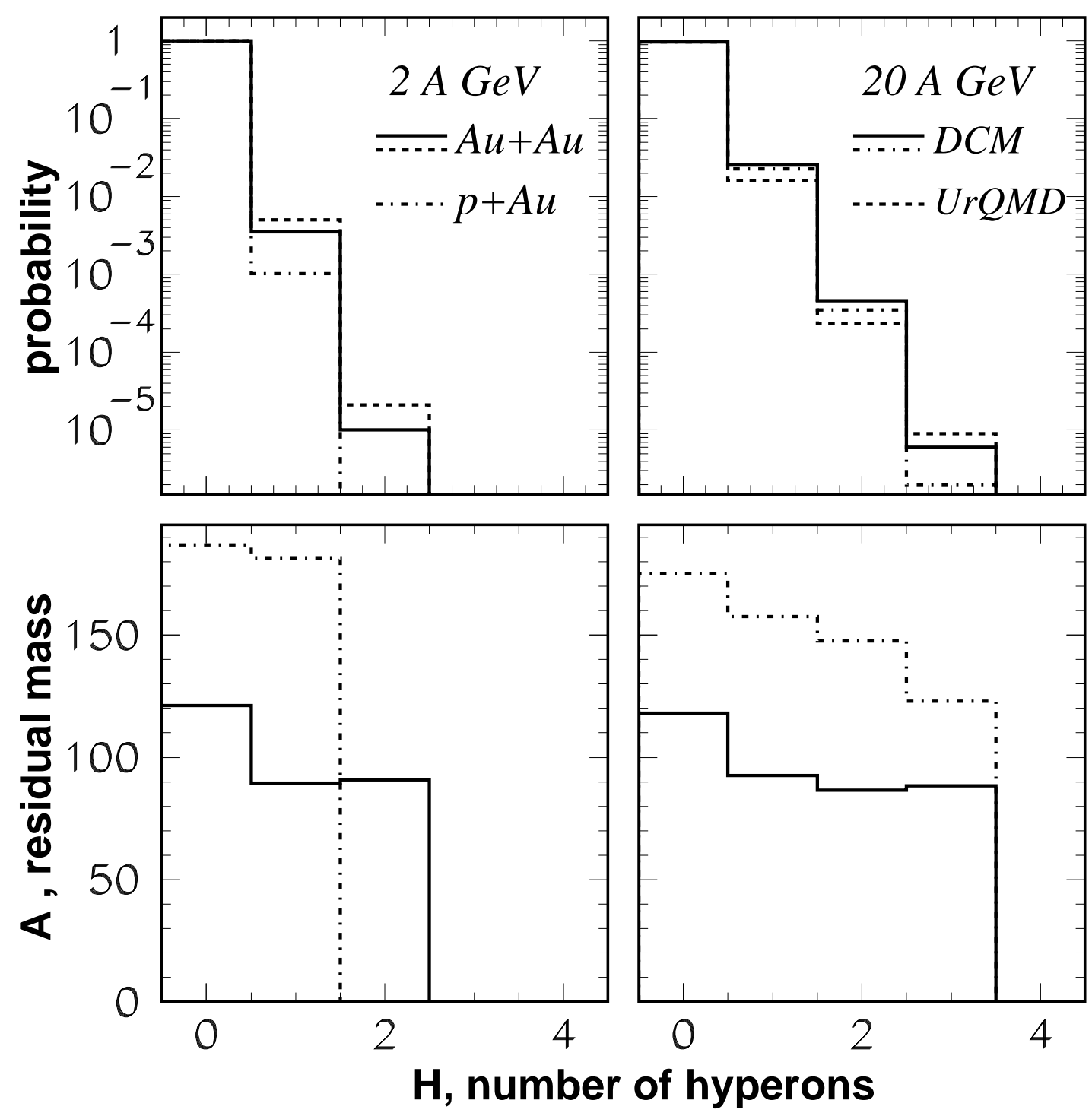

FIG. 6: Probability for formation of conventional and strange spectator residuals (top panels), and their mean mass numbers (bottom panels) versus the number of captured $\Lambda$ hyperons $(\mathrm{H})$, calculated with DCM and UrQMD model for $\mathrm{p}+\mathrm{Au}$ and $\mathrm{Au}+\mathrm{Au}$ collisions with energy of 2 $\mathrm{GeV}$ per nucleon (left panels), and $20 \mathrm{GeV}$ per nucleon (right panels). The reactions and energies are noted in the figure by different histograms. 


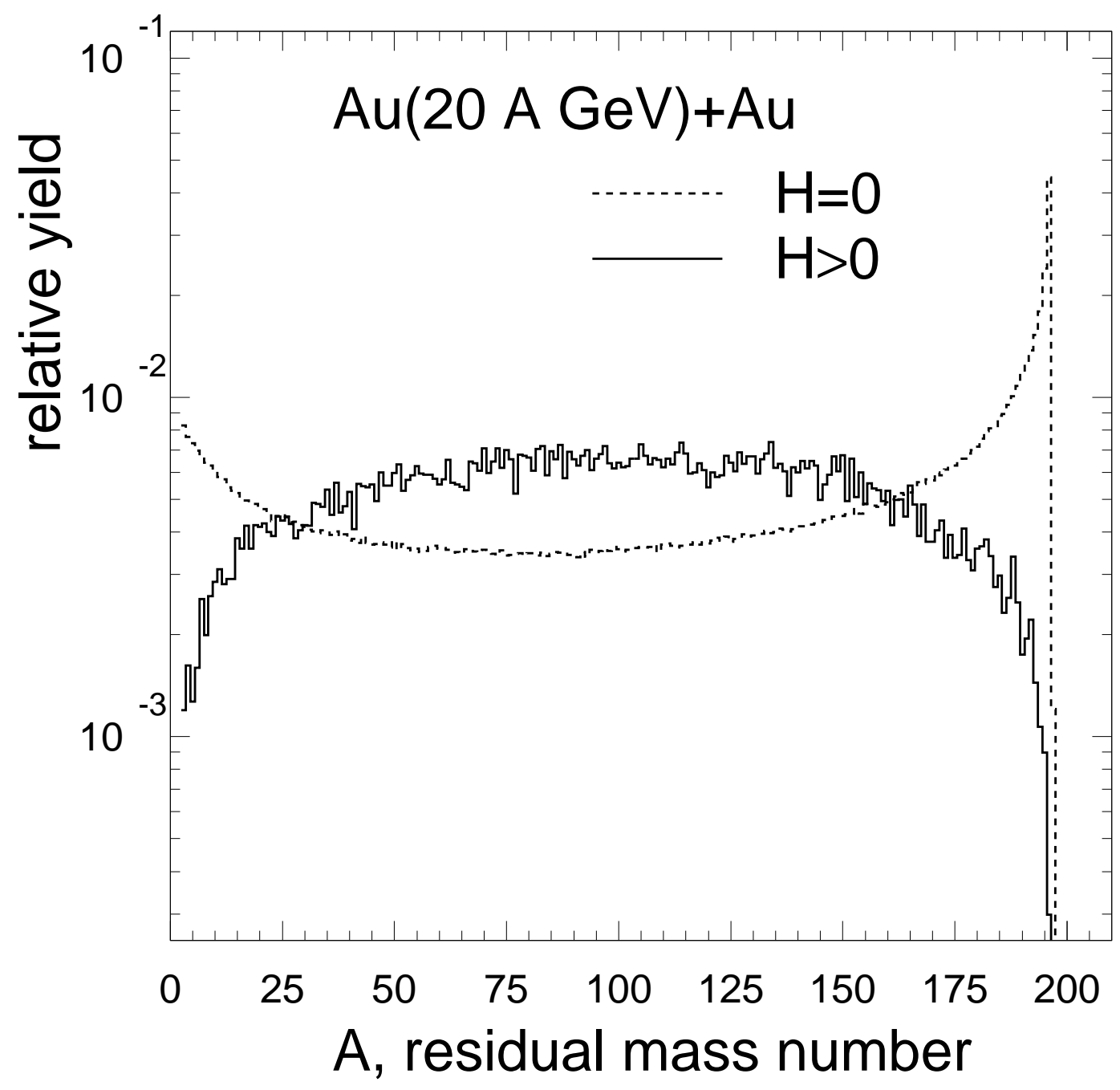

FIG. 7: Mass distribution of conventional $(\mathrm{H}=0)$ and hypernuclear $(\mathrm{H}>0)$ spectators after DCM calculations for $\mathrm{Au}+\mathrm{Au}$ collisions with projectile energy $20 \mathrm{GeV}$ per nucleon. 


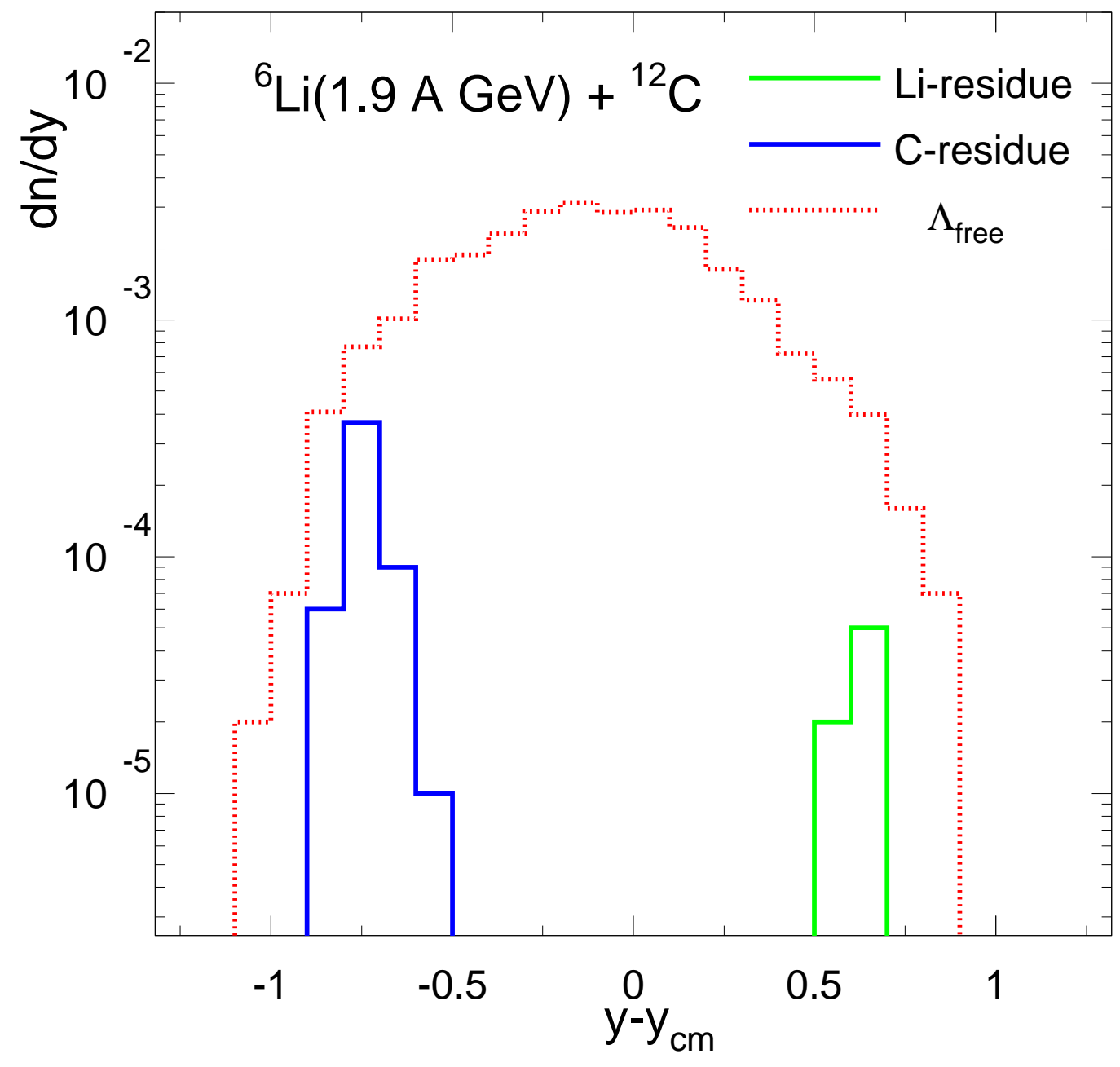

FIG. 8: DCM calculations of rapidity distribution for free $\Lambda$ and for hyper-residues with one $\Lambda$ coming from target $(\mathrm{C})$ and projectile $(\mathrm{Li})$ spectators in reaction ${ }^{6} \mathrm{Li}(1.9 \mathrm{~A} \mathrm{GeV})+{ }^{12} C$. 


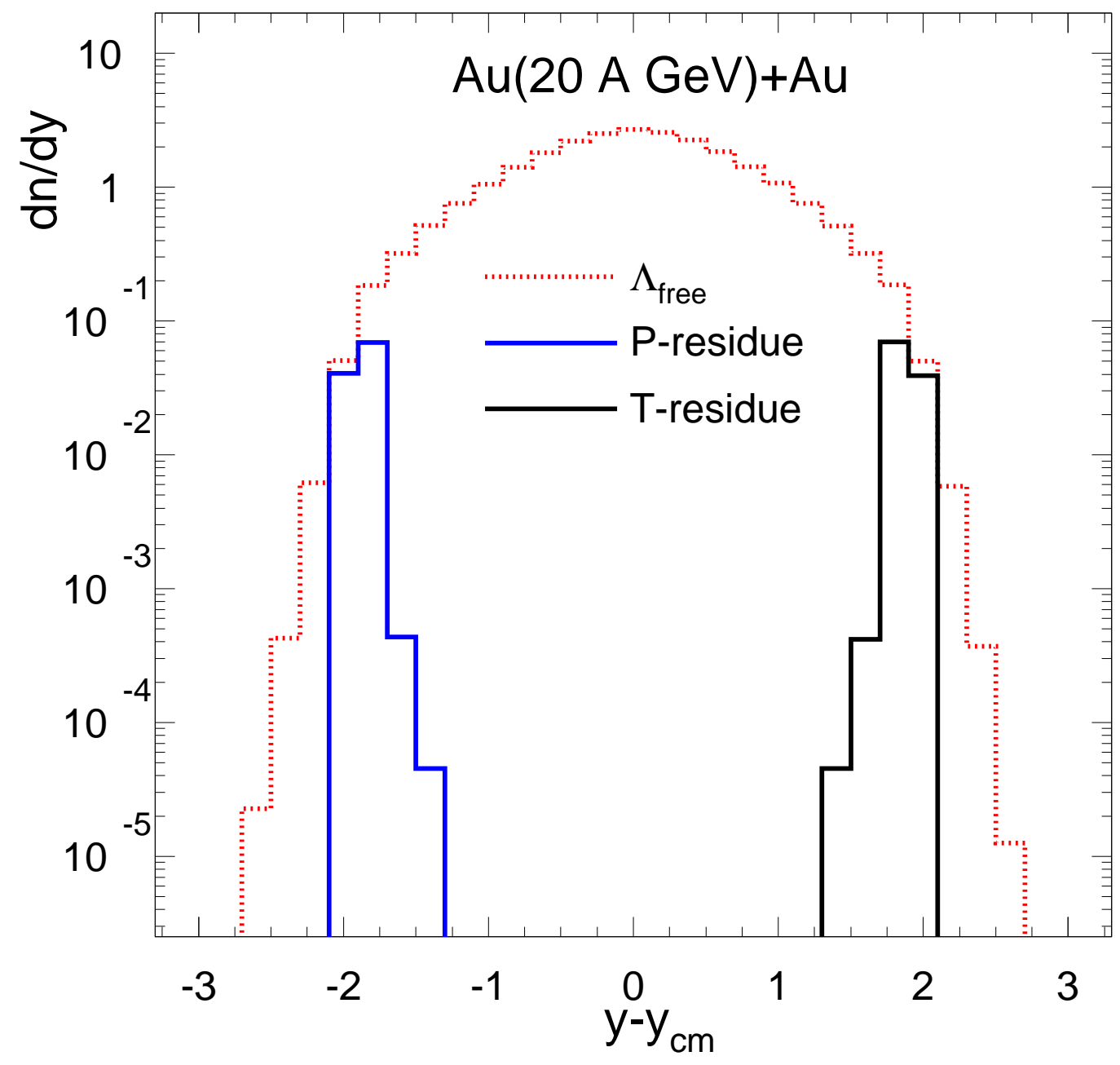

FIG. 9: DCM calculations of rapidity distribution for free $\Lambda$ and for hyper-residues coming from target $(\mathrm{T})$ and projectile $(\mathrm{P})$ spectators in the reaction ${ }^{197} \mathrm{Au}(20 \mathrm{~A} \mathrm{GeV})+{ }^{197} \mathrm{Au}$. 


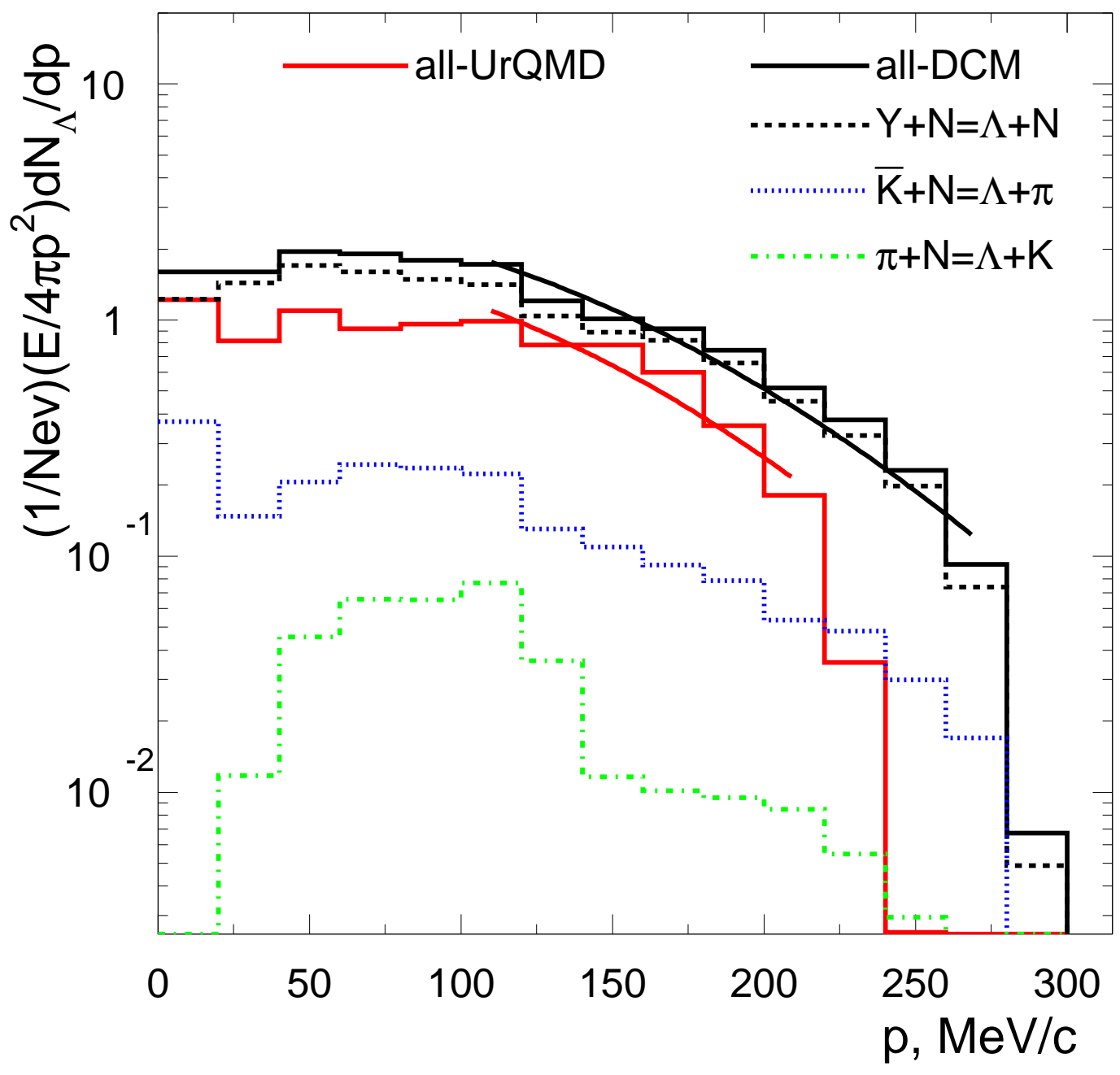

FIG. 10: Momentum distribution of $\Lambda$ hyperons captured in the spectators. Notations are the same as in Fig. 4. Solid curves show the Boltzmann fits of the distributions with temperature $\sim 10$ $\mathrm{MeV}$. 